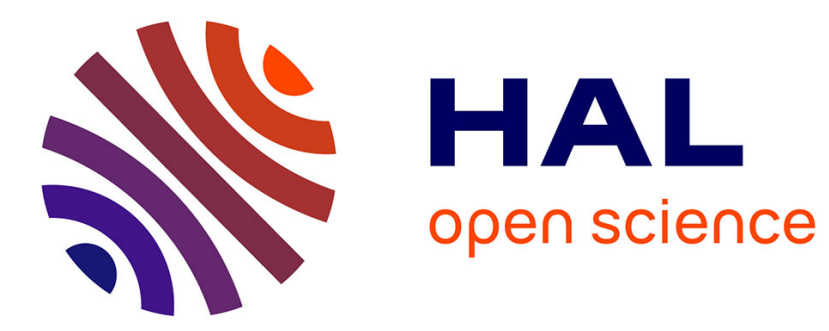

\title{
Le secret sur la torture pendant la guerre d'Algérie
} Raphaëlle Branche, Sylvie Thénault

\section{To cite this version:}

Raphaëlle Branche, Sylvie Thénault. Le secret sur la torture pendant la guerre d'Algérie. Matériaux pour l'histoire de notre temps, 2000, Le secret en histoire, 58, pp.57-63. 10.3406/mat.2000.404251. hal-02355862

\section{HAL Id: hal-02355862 \\ https://hal.science/hal-02355862}

Submitted on 8 Nov 2019

HAL is a multi-disciplinary open access archive for the deposit and dissemination of scientific research documents, whether they are published or not. The documents may come from teaching and research institutions in France or abroad, or from public or private research centers.
L'archive ouverte pluridisciplinaire HAL, est destinée au dépôt et à la diffusion de documents scientifiques de niveau recherche, publiés ou non, émanant des établissements d'enseignement et de recherche français ou étrangers, des laboratoires publics ou privés.

\section{(이) $\$$}

Distributed under a Creative Commons Attribution - NonCommercial - NoDerivatives $\mid 4.0$ 


\section{Le secret sur la torture pendant la guerre d'Algérie}

Madame Sylvie Thenault, Madame Raphaele Branche

\section{Citer ce document / Cite this document :}

Thenault Sylvie, Branche Raphaele. Le secret sur la torture pendant la guerre d'Algérie. In: Matériaux pour l'histoire de notre temps, $n^{\circ} 58,2000$. Le secret en histoire. pp. 57-63;

doi : https://doi.org/10.3406/mat.2000.404251

https://www.persee.fr/doc/mat_0769-3206_2000_num_58_1_404251

Fichier pdf généré le 30/03/2018 


\section{Le secret sur la torture pendant la guerre d'Algérie}

$\mathrm{L}$ es forces de l'ordre chargées, en Algérie, de réprimer la rébellion entre 1954 et 1962 ont pratiqué la torture : cette réalité est aujourd'hui connue. Elle l'était déjà à l'époque, la presse s'étant fait notamment l'écho des dénonciations des sévices subis, principalement, par les Algériens.

S'il n'y a donc pas à proprement parler de secret sur la torture pendant la guerre d'Algérie, il y a cependant des révélations régulières qui signalent la persistance d'un doute sur cette réalité : étaitelle massive ou n'était-elle qu'exceptionnelle? Était-elle encouragée par le pouvoir? Les appelés, Enfants des lecteurs des journaux métropolitains, Q $=$ taient-ils amenés à la pratiquer? De nombreuses Ğuestions sont en fait restées en suspens pendant la guerre et toutes les réponses apportées n'ont 1 doc s réussi à résorber cette impression d'un secret sur le sujet.

- Le secret existe en effet bien sur la torture penc'ant la guerre d'Algérie. Il est le résultat de multiples constructions que nous allons essayer de présenter ici. Elles peuvent être ramassées en deux \&rands ensembles : les stratégies de dissimulation tes stratégies de divulgation.

\section{Les stratégies de dissimulation : garder la torture secrète}

La volonté de garder la torture secrète est bien à l'œuvre pendant la guerre d'Algérie puisque la pratique de la torture est interdite en France comme en Algérie entre 1954 et 1962. Même dans le cadre d'une guerre - ce qui n'est pas le cas, officiellement, des événements en question , elle est interdite, au moins depuis les conventions de Genève ${ }^{1}$. Les militaires comme les policiers qui y ont recours sont passibles de condamnations pénales et disciplinaires. Ils ont donc tout intérêt à garder le secret sur cette pratique.

Le visage terroriste de la guerre d'Algérie a cependant amené certains chefs militaires et politiques à considérer la torture comme un moindre mal face aux ennemis de la France. Mais l'opinion publique, tenue informée par une presse libre malgré des censures et des saisies -, reste, à peine dix ans après la fin de la Seconde Guerre mondiale, sensible sur ce point. Pour les exécutants, le secret sur cette pratique s'impose donc absolument.

C'est à l'étude de la préservation de ce secret que nous allons dans un premier temps nous consacrer; le meilleur moyen de garder une pratique secrète étant de n'en laisser aucune trace ce qui, pour l'historien, n'est pas sans poser des problèmes... Deux types de traces sont évités : les traces écrites et les traces physiques.

Une armée a toujours intérêt à ce que la discipline règne dans ses rangs. Pour cette raison, elle ne peut laisser les soldats, ou même les chefs d'unité, libres de tous leurs gestes et décisions. La manière dont on fait la guerre relève bien du haut commandement et pas simplement des exécutants : les responsables militaires ont dû prendre position sur la question de la torture. Quand on étudie les directives, les ordres et autres circulaires produits par les plus hauts responsables de la guerre d'Algérie, on repère une attention extrême aux termes employés. Dans certains textes, les mots " torture » et "sévices " sont utilisés mais il $s^{\prime}$ agit toujours alors d'instructions les interdisant. Informés de la réalité de certains sévices, les responsables, auteurs de ce type de textes, précisent que ces pratiques sont illégales et seront à l'avenir sévèrement sanctionnées. On apprend ainsi que les responsables civils et militaires étaient au courant de ces pratiques, mais aussi qu'ils les condamnaient.

Par contre, dans les textes officiels sur les méthodes de répression à employer, jamais le mot de " torture " n'est utilisé. Est-ce à dire que la pratique n'est jamais préconisée? Pour se faire comprendre, les auteurs ont en fait recours à un certain nombre de subterfuges allant de l'euphémisation à la métaphore. Très vite un lexique parallèle se met en fait en place qui permet de décrypter, sans trop de doute possible, la réalité des consignes données, tout en laissant aussi toujours la possibilité à leurs auteurs de nier avoir donné explicitement - et c'est tout le problème, mais c'est le seul problème - de tels ordres.

Ainsi, pendant la bataille d'Alger, quand le général Salan, dans une note de service très secrète adressée à tous les commandants de Corps d'Armée, écrit : " de récentes expériences effectuées dans certains régions ont mis en lumière le parti qui pouvait être tiré, surtout dans les villes, d'interrogatoires poussés à fond et immédiatement exploités", et qu'il ajoute " tout individu appréhendé [doit être] soumis à un interrogatoire aussi serré que possible ${ }^{2}{ }^{2}$, on peut lire qu'il recommande les méthodes utilisées par les hommes du général Massu à Alger, à savoir notamment la torture. "Interrogatoire » est en effet devenu le "synonyme légal " ${ }^{3}$ de torture : le mot se décline en "interrogatoire sous la contrainte ", " interrogatoire contraint ", " interrogatoire musclé " mais il désigne toujours la même réalité.

Ceci dit, l'usage d'expressions au sens légèrement décalé laisse une marge d'interprétation aux
1. Le traitement humain des prisonniers est théoriquement garanti par l'article 13 de la convention et précisé à l'article 17 par les alinéas c et $d$, le premier interdisant les interrogatoires inhumains ( $c^{\prime}$ est-à-dire toute forme de contrainte physique ou morale visant à arracher des aveux ou obtenir la collaboration du prisonnier contre sa volonté) et le second toute forme d'humiliation.

2. Note de service du 11 mars 1957, 1H 3087/1 (SHAT, Service Historique de I'Armée de Terre).

3. Gabriel Périès,

"Conditions d'emploi des termes interrogatoire et torture dans le discours militaire pendant la guerre d'Algérie ", Mots, n' 51 , juin 1997, pp. 41-57. 
destinataires des textes : marge qui restitue toute sa place à l'oralité. Les instructions, directives et autres textes réglementaires sont en effet amenés à être diffusés jusqu'aux échelons inférieurs et commentés par les responsables subalternes. C'est ce commentaire qui est le véritable ordre pour le soldat, et il est oral.

L'importance de l'oralité pour l'expression de I'autorité est bien connue. Par exemple, quand le général Salan, en avril 1957, veut empêcher que se reproduisent des crimes perpétrés par des militaires français sur les populations désarmées, il adresse une lettre aux commandants de Corps d'Armée en leur demandant d'intervenir personnellement très fermement : mieux qu'une instruction du général en chef, il sait qu'une intervention personnelle des commandants locaux est le meilleur moyen de voir sa volonté traduite en actes $^{4}$

Il ne s'agit pas de dire que les ordres verbaux encouragent systématiquement des formes illégales de répression, tandis que les ordres écrits l'interdiraient. Néanmoins, il est évident que les ordres oraux offrent une latitude beaucoup plus grande aux responsables militaires sur le terrain. Le général Salan précise d'ailleurs en bas de sa note d'avril 1957 adressée aux commandants de Corps d'Armée et recommandant les interrogatoires serrés, qu'elle doit être diffusée uniquement oralement aux subordonnés : les traces de l'instruction du général en chef doivent s'arrêter au niveau supérieur de la hiérarchie. En outre, la place importante laissée à l'oralité dans la guerre est renforcée par les moyens de communication modernes (tels que le téléphone et la radio) : des ordres importants peuvent être donnés oralement et appliqués rapidement.

Ainsi, le secret est bien construit à l'échelon supérieur. Qu'en est-il à celui des exécutants? Comment, au plus près de la pratique, est élaboré le secret sur la torture? Bien souvent, le secret sur les opérations menées est considéré comme une condition sine qua non de la réussite. Évidente dans les guerres classiques, cette considération est $d^{\prime}$ autant plus vraie dans une guerre de renseignements comme celle que mènent certains services ou certains soldats en Algérie. "Aucun ordre écrit ne sera donné " précise ainsi une note de service de juillet 1958 à propos d'une opération ${ }^{5}$. II est toutefois précisé par écrit aux exécutants leur marge de manœuvre : " à l'issue de l'opération ou même en cours d'opération, des décisions immédiates doivent être prises vis-à-vis des personnes arrêtées et reconnues coupables. [...] Certaines doivent être abattues par la troupe opérationnelle. D'autres doivent être internées ". Toutes les décisions particulières quant aux individus ou quant au détail de l'opération, sont donc du domaine de l'oral : l'écrit fixant uniquement le cadre pour les exécutants. Bien que cet exemple relève d'un service spécial, le rapport qu'y entretiennent l'oral et

Le soldat est donc toujours confronté à des ordres oraux - qui ne laissent aucune trace directe pour I'historien. La force d'un ordre oral est théoriquement moins forte que celle d'un ordre écrit puisqu'un soldat peut demander la confir- mation par écrit d'un ordre oral. Dans les faits, de tels actes sont rares $^{6}$ : les soldats n'étant pas informés de leurs droits et surtout n'étant pas toujours en situation de les faire valoir. Les hésitations des soldats sont de peu de poids face à la force des ordres donnés.

Le secret de la torture n'existe donc pas à proprement parler puisque tous les militaires qui ont été au contact de cette réalité - et ils sont nombreux - en connaissent l'existence. Leurs témoignages sont d'ailleurs une des sources principales tant à l'époque qu'aujourd'hui. Les responsables de la guerre ont néanmoins eu un souci, croissant au cours de la guerre : éviter de laisser des traces de cette pratique. En ce sens et pour l'histoire, il y a bien eu une tentative de construction d'un secret de la torture pendant la guerre d'Algérie.

Ce secret n'a pas été élaboré a posteriori par destruction des preuves et des écrits (même si de telles démarches ne sont pas à exclure), il l'a été dès l'époque. On a vu la prudence dans l'expression et le jeu entre l'écrit et l'oral qui ont permis de faire coexister au moins deux discours au sein de l'institution militaire. Cette conscience des traces se retrouve aussi quand on étudie la manière même dont les "interrogatoires sous la contrainte " étaient menés : le souci de ne pas laisser de traces physiques apparaît nettement.

Dans une causerie sur le renseignement destinée aux officiers en stage en Algérie dans les dernières années de la guerre, il est expliqué aux stagiaires que lorsque l'Officier de Renseignement - qui est l'officier chargé des interrogatoires au sein des secteurs ou des unités militaires - leur demandera de lui envoyer un prisonnier fait au combat, celui-ci devra lui être transféré " rapidement, intact et avec les documents saisis sur lui ${ }^{7}$. " Intact » est explicité ainsi : " attention aux harkis, à leur brutalité possible et aux questions inopportunes ». Ce souci de l'intégrité physique des prisonniers n'est pas nouveau. En 1957, le général Allard ${ }^{8}$ proscrivait déjà, notamment au $3^{\mathrm{e}} \mathrm{RPC}$, " tout procédé qui marquerait irrémédiablement l'individu moralement ou physiquement " 9 . II réitère d'ailleurs cet ordre en 1959.

L'usage de l'électricité pour faire parler des suspects ou des prisonniers a pu être vanté pour cette raison : contrairement à d'autres sévices, cette méthode - aussi efficace que d'autres - ne laissait pas de séquelles durables. Les réserves de certains militaires sur ce point peuvent être rattachées à des considérations éthiques. Il faut aussi se souvenir que certains nationalistes, inculpés de participation à la rébellion, déclarent que les aveux obtenus d'eux l'avaient été sous la torture. Or, devant la justice, les traces de ces tortures sont les seules preuves qu'ils pouvaient alléguer. Sans traces visibles qu'un médecin peut constater, il n'y a pas eu de torture.

Paul Thibaud, dans un article paru en mai 1957 dans la revue Esprit ${ }^{10}$, se fait l'écho de cette disparition des traces. Évoquant les tortures à l'électricité, il écrit : "I'application des électrodes sur une peau mouillée (par la sueur par exemple) risque seulement de provoquer des étincelles et par conséquent de petites brûlures ». II ajoute : " ces lésions ont d'ailleurs souvent été constatées l'écrit est généralisable à d'autres situations.

\footnotetext{
6. On connaît le cas du
sous-lieutenant Jean le
Meur mis aux arrêts de
rigueur pour avoir critiqué
un ordre entendu par
radio de son commandant
d'unité ordonnant de ne
pas faire de prisonniers
(Témoignages et Docu-
ments, n' 19, décembre
1959).
7. Causerie destinée aux
officiers stagiaires du
CICPG d'Arzew sur le
renseignement en Algérie,
postérieure au printemps
1960, 1H 1485 (SHAT).
8. Commandant du Corps
d'Armée d'Alger.
9. Instruction du 27 mars
1957, 1H 2698/2 (SHAT).
10. Paul Thibaud, "Com-
ment fonctionne la justice
en Algérie ", Esprit, mai
1957.

4. Lettre du général Sala
aux commandants de
Corps d'Armée, 27 avril
1957, $1 \mathrm{H} 2579 / 2$ (SHA $)$ 6. On connaît le cas du
sous-lieutenant Jean le
Meur mis aux arrêts de
rigueur pour avoir critiqué
un ordre entendu par
radio de son commandant
d'unité ordonnant de ne
pas faire de prisonniers
(Témoignages et Docu-
ments, n' 19, décembre
1959).
7. Causerie destinée aux
officiers stagiaires du
CICPG d'Arzew sur le
renseignement en Algérie,
postérieure au printemps
1960, 1H 1485 (SHAT).
8. Commandant du Corps
d'Armée d'Alger.
9. Instruction du 27 mars
1957, 1H 2698/2 (SHAT).
10. Paul Thibaud, "Com-
ment fonctionne la justice
en Algérie ", Esprit, mai
1957.

6. On connaît le cas du
sous-lieutenant Jean le
Meur mis aux arrêts de
rigueur pour avoir critiqué
un ordre entendu par
radio de son commandant
d'unité ordonnant de ne
pas faire de prisonniers
(Témoignages et Docu-
ments, n' 19, décembre
1959).
7. Causerie destinée aux
officiers stagiaires du
CICPG d'Arzew sur le
renseignement en Algérie,
postérieure au printemps
1960, 1H 1485 (SHAT).
8. Commandant du Corps
d'Armée d'Alger.
9. Instruction du 27 mars
1957, 1H 2698/2 (SHAT).
10. Paul Thibaud, "Com-
ment fonctionne la justice
en Algérie ", Esprit, mai
1957.

6. On connaît le cas du
sous-lieutenant Jean le
Meur mis aux arrêts de
rigueur pour avoir critiqué
un ordre entendu par
radio de son commandant
d'unité ordonnant de ne
pas faire de prisonniers
(Témoignages et Docu-
ments, n' 19, décembre
1959).
7. Causerie destinée aux
officiers stagiaires du
CICPG d'Arzew sur le
renseignement en Algérie,
postérieure au printemps
1960, 1H 1485 (SHAT).
8. Commandant du Corps
d'Armée d'Alger.
9. Instruction du 27 mars
1957, 1H 2698/2 (SHAT).
10. Paul Thibaud, "Com-
ment fonctionne la justice
en Algérie ", Esprit, mai
1957. 5. Note de la section

1958, 1H3087/1 (SHAT).
6. On connaît le cas du
sous-lieutenant Jean le
Meur mis aux arrêts de
rigueur pour avoir critiqué
un ordre entendu par
radio de son commandant
d'unité ordonnant de ne
pas faire de prisonniers
(Témoignages et Docu-
ments, n' 19, décembre
1959).
7. Causerie destinée aux
officiers stagiaires du
CICPG d'Arzew sur le
renseignement en Algérie,
postérieure au printemps
1960, 1H 1485 (SHAT).
8. Commandant du Corps
d'Armée d'Alger.
9. Instruction du 27 mars
1957, 1H 2698/2 (SHAT).
10. Paul Thibaud, "Com-
ment fonctionne la justice
en Algérie ", Esprit, mai
1957.

6. On connaît le cas du
sous-lieutenant Jean le
Meur mis aux arrêts de
rigueur pour avoir critiqué
un ordre entendu par
radio de son commandant
d'unité ordonnant de ne
pas faire de prisonniers
(Témoignages et Docu-
ments, n' 19, décembre
1959).
7. Causerie destinée aux
officiers stagiaires du
CICPG d'Arzew sur le
renseignement en Algérie,
postérieure au printemps
1960, 1H 1485 (SHAT).
8. Commandant du Corps
d'Armée d'Alger.
9. Instruction du 27 mars
1957, 1H 2698/2 (SHAT).
10. Paul Thibaud, "Com-
ment fonctionne la justice
en Algérie ", Esprit, mai
1957.

6. On connaît le cas du
sous-lieutenant Jean le
Meur mis aux arrêts de
rigueur pour avoir critiqué
un ordre entendu par
radio de son commandant
d'unité ordonnant de ne
pas faire de prisonniers
(Témoignages et Docu-
ments, n' 19, décembre
1959).
7. Causerie destinée aux
officiers stagiaires du
CICPG d'Arzew sur le
renseignement en Algérie,
postérieure au printemps
1960, 1H 1485 (SHAT).
8. Commandant du Corps
d'Armée d'Alger.
9. Instruction du 27 mars
1957, 1H 2698/2 (SHAT).
10. Paul Thibaud, "Com-
ment fonctionne la justice
en Algérie ", Esprit, mai
1957.

6. On connaît le cas du
sous-lieutenant Jean le
Meur mis aux arrêts de
rigueur pour avoir critiqué
un ordre entendu par
radio de son commandant
d'unité ordonnant de ne
pas faire de prisonniers
(Témoignages et Docu-
ments, n' 19, décembre
1959).
7. Causerie destinée aux
officiers stagiaires du
CICPG d'Arzew sur le
renseignement en Algérie,
postérieure au printemps
1960, 1H 1485 (SHAT).
8. Commandant du Corps
d'Armée d'Alger.
9. Instruction du 27 mars
1957, 1H 2698/2 (SHAT).
10. Paul Thibaud, "Com-
ment fonctionne la justice
en Algérie ", Esprit, mai
1957.

6. On connaît le cas du
sous-lieutenant Jean le
Meur mis aux arrêts de
rigueur pour avoir critiqué
un ordre entendu par
radio de son commandant
d'unité ordonnant de ne
pas faire de prisonniers
(Témoignages et Docu-
ments, n' 19, décembre
1959).
7. Causerie destinée aux
officiers stagiaires du
CICPG d'Arzew sur le
renseignement en Algérie,
postérieure au printemps
1960, 1H 1485 (SHAT).
8. Commandant du Corps
d'Armée d'Alger.
9. Instruction du 27 mars
1957, 1H 2698/2 (SHAT).
10. Paul Thibaud, "Com-
ment fonctionne la justice
en Algérie ", Esprit, mai
1957.

6. On connaît le cas du
sous-lieutenant Jean le
Meur mis aux arrêts de
rigueur pour avoir critiqué
un ordre entendu par
radio de son commandant
d'unité ordonnant de ne
pas faire de prisonniers
(Témoignages et Docu-
ments, n' 19, décembre
1959).
7. Causerie destinée aux
officiers stagiaires du
CICPG d'Arzew sur le
renseignement en Algérie,
postérieure au printemps
1960, 1H 1485 (SHAT).
8. Commandant du Corps
d'Armée d'Alger.
9. Instruction du 27 mars
1957, 1H 2698/2 (SHAT).
10. Paul Thibaud, "Com-
ment fonctionne la justice
en Algérie ", Esprit, mai
1957.

6. On connaît le cas du
sous-lieutenant Jean le
Meur mis aux arrêts de
rigueur pour avoir critiqué
un ordre entendu par
radio de son commandant
d'unité ordonnant de ne
pas faire de prisonniers
(Témoignages et Docu-
ments, n' 19, décembre
1959).
7. Causerie destinée aux
officiers stagiaires du
CICPG d'Arzew sur le
renseignement en Algérie,
postérieure au printemps
1960, 1H 1485 (SHAT).
8. Commandant du Corps
d'Armée d'Alger.
9. Instruction du 27 mars
1957, 1H 2698/2 (SHAT).
10. Paul Thibaud, "Com-
ment fonctionne la justice
en Algérie ", Esprit, mai
1957.

6. On connaît le cas du
sous-lieutenant Jean le
Meur mis aux arrêts de
rigueur pour avoir critiqué
un ordre entendu par
radio de son commandant
d'unité ordonnant de ne
pas faire de prisonniers
(Témoignages et Docu-
ments, n 19, décembre
1959).
7. Causerie destinée aux
officiers stagiaires du
CICPG d'Arzew sur le
renseignement en Algérie,
postérieure au printemps
1960, 1H 1485 (SHAT).
8. Commandant du Corps
d'Armée d'Alger.
9. Instruction du 27 mars
$1957,1 H 2698 / 2$ (SHAT).
10. Paul Thibaud, "Com-
ment fonctionne la justice
en Algérie ", Esprit, mai
1957.
} 
par les médecins, mais on les attribue officiellement à des "maladies de peau», à "une épidémie d'eczéma due au climat nord-africain» ». II conclut : "D'après le Dr Hovnanian "1, cette forme d'eczéma serait particulière aux prisons d'Algérie ".

Les témoignages sur les lieux de torture prouvent que les militaires français qui recourent à de tels procédés ont conscience de cette dimension. Les prisonniers trop abîmés sont souvent gardés au secret en attendant qu'ils se remettent ou soignés dans les lieux mêmes où ils ont été interrogés. Certains sont aussi exécutés sommairement à la suite d'interrogatoires trop violents. Dans les deux cas, il s'agit de détruire ou d'effacer les traces visibles des tortures infligées aux prisonniers : le secret est ainsi préservé.

On pourrait penser que les Algériens ont la possibilité de parler et de raconter les sévices, que ceux-ci aient ou non laissé des traces, mais dans un contexte et sur un sujet où la parole d'un Algérien n'a pas de poids, c'est bien la trace matérielle qui seule peut faire foi. Les rapports d'expertise médicale sont en fait les seules preuves acceptées de ces pratiques - d'où des discussions parfois serrées sur ces rapports.

L'illégalité de la pratique de la torture était connue de tous les militaires engagés en Algérie. Selon les contextes opérationnels, selon la personnalité des hommes, selon aussi la distance physique avec les interrogatoires, les réactions à cette pratique ont été très différentes. Une entreprise générale de dissimulation a pu être décelée mais s'agissait-il, à tous les niveaux, de la même dissimulation? En fait, parallèlement à la pyramide hiérarchique, on peut sans doute dessiner une pyramide de secrets emboîtés ${ }^{12}$ et distinguer grossièrement deux niveaux : celui des exécutants et celui de leurs supérieurs.

Les exécutants ont, en général, le souci de dissimuler leurs pratiques. Les témoignages sont nombreux des prisonniers déplacés lors des visites de membres de commissions d'enquête ou d'inspection, qu'ils soient militaires ou civils, français ou étrangers. II est aussi facile de faire disparaître les installations utilisées pour les tortures, ou de les rendre rapidement à leur usage légal pour les génératrices de campagne.

Quant aux autorités supérieures, que ce soient les supérieurs hiérarchiques ou les autorités civiles, il est difficile de savoir de quelles informations elles disposent exactement. Mais la torture n'est un secret pour personne. On sait même que certains ministres ont encouragé verbalement le général Massu et ses méthodes au cours de la bataille $d^{\prime}$ Alger ${ }^{13}$. Si presque tous les responsables politiques et militaires tiennent pourtant à dissimuler aux yeux de l'opinion l'existence de cette pratique dans les rangs des forces de l'ordre, s'ils tentent de maintenir le secret sur la torture, c'était qu'ils dissimulent aussi ainsi leur impuissance à en contrôler l'interdiction.

Malgré toutes ces précautions pour dissimuler la pratique de la torture, l'opinion publique a, à de nombreuses reprises, été informée de son existence. Comment s'est opérée cette divulgation du secret? C'est l'objet de notre seconde partie, qui se concentre sur les premières années de la guerre (et notamment sur l'année 1957) qui voient la révélation au grand jour de cette pratique ${ }^{14}$.

\section{Les stratégies de divulgation ou le temps de la révélation du secret}

Mettre à jour les mécanismes de la divulgation de la pratique de la torture revient à reconstituer la chaîne de la victime jusqu'à la presse ou l'éditeur assurant la publicité de l'affaire. Trois cercles agissant les uns après les autres se dégagent : ceux qui savent; ceux qui s'engagent; ceux qui publient.

Les premiers à savoir que la torture est pratiquée sont évidemment les victimes elles-mêmes. Leurs témoignages sont la plupart du temps constitués par le texte des plaintes qu'elles ont déposées. La plainte présente l'avantage d'attester la crédibilité des faits dans la mesure où ils sont soumis à la vérification de la justice. Or, dans un contexte de dissimulation de la pratique de la torture, l'exigence de crédibilité du témoignage est redoublée. Le livre d'Henri Alleg, La question, constitue une exception notable $d^{\prime}$ expression directe par une victime. Ce cas unique peut s'expliquer par le fait qu'Henri Alleg est un professionnel de l'écriture puisqu'il a dirigé le journal Alger Républicain. Son témoignage est $d^{\prime}$ 'une force rare et, un mois après sa parution en février 1958 aux éditions de Minuit, au moment où il est saisi, plusieurs dizaines de milliers d'exemplaires en ont déjà été vendus.

En contact avec les victimes qu'ils incitent à porter plainte, les avocats sont les principaux informateurs de la presse. Pierre Stibbe est ainsi à l'origine des deux premiers articles dénonçant la torture en Algérie, parus en janvier 1955 : "Votre Gestapo d'Algérie ", de Claude Bourdet dans France-Observateur et "La question » de François Mauriac dans l'Express ${ }^{15}$. De plus, pour les avocats, faire connaître les tortures subies par leurs clients peut s'inscrire dans le cadre d'une stratégie de défense, en particulier au moment de plaider la grâce des condamnés à mort. La première affaire dans laquelle s'est engagé Jacques Vergès en fournit un bon exemple : Djamila Bouhired est condamnée à mort en juillet 1957 et, deux mois plus tard, paraît Pour Djamila Bouhired de Georges Arnaud, aux éditions de Minuit, point de départ de la campagne pour la grâce de la jeune fille.

Enfin, certains fonctionnaires d'Algérie en contact avec les victimes, comme les magistrats ou le personnel des préfectures chargé de la gestion des centres d'internement, appartiennent eux aussi au cercle de ceux qui savent avec certitude, sans contestation possible, que la torture est pratiquée. Mais leur profession, qui les met au service de l'État, leur interdit toute publicité même quand la pratique de la torture, qu'ils constatent et réprouvent, leur pose un cas de conscience. En 1957, Jean Reliquet, procureur général d'Alger, qui visite la prison d'Alger et fait recueillir des plaintes par son substitut, ainsi que Paul Teitgen, secrétaire général de la préfecture d'Alger, lors de son inspection du camp de Paul-Cazelles, ont été confrontés à cette situation insoutenable. Ils font
11. Membre de la commission parlementaire chargée d'enquêter sur les sévices commis sur des communistes algériens à Oran en septembre 1956, le Dr Hovnanian avait préféré s'abstenir sur le rapport final (rapport Provo) arguant " d'un ensemble de faits, en partie médicaux, lui ayant paru probants de méthodes policières incompatibles avec la véritable mission de la police ".

12. L'aspect systématique de cet article ne doit cependant jamais occulter l'extrême diversité des situations qui coexistent pendant la guerre d'Algérie, en particulier sur la question de la torture. 13. Rapport du général Massu sur la bataille d'Alger, 5 juin 1957, 304 AP 701/1 (fonds Garçon, (AC).

14. Cette première partie a été rédigée par Raphaëlle Branche, la seconde partie par Sylvie Thénault. 15. Parus respectivement le 13 et le 15 janvier 1955. 
alors le choix de faire circuler l'information sans pour autant la rendre publique. IIs informent en effet les membres des commissions d'enquête envoyés par la métropole, comme Maurice Garçon de la Commission de sauvegarde et Louis Martin-Chauffier de la Commission internationale contre le régime concentrationnaire ${ }^{16}$. Ils participent donc à la divulgation de l'information mais en restant dans l'ombre et en se couvrant puisqu'ils ne parlent qu'à des Commissions officielles. Du fait de leur statut professionnel, ils ne mettent en œuvre qu'une divulgation réfléchie et maîtrisée, en prenant le soin de ne pas alimenter la campagne de presse qui secoue la métropole au moment de la "bataille d'Alger".

Le cercle de ceux qui s'engagent comprend ceux qui mettent leur notoriété ou leurs réseaux d'information au service d'une affaire de torture. Le plus souvent, au départ de l'affaire, joue de façon primordiale un lien personnel entre la victime et celui qui se mobilise pour défendre sa cause. En effet, en l'absence de preuves indiscutables et face à la négation de la pratique de la torture, le doute et l'incrédulité doivent être vaincus. L'engagement sur une affaire nécessite une relation de confiance entre la victime et la personnalité qui entreprend la médiatisation de son cas.

Deux des affaires célèbres qui ont mobilisé les milieux universitaires se plient à cette règle. C'est d'abord le cas pour Ali Boumendjel, arrêté à Alger en février 1957, détenu par les parachutistes et dont le suicide est officiellement annoncé au début du mois de mars. René Capitant, professeur de droit dont Ali Boumendjel a été l'élève, choisit alors de suspendre ses cours et sa réaction n'est que le début d'une cascade de protestations. Le cas de Maurice Audin, arrêté en juin 57 par les parachutistes à Alger, disparu, présente des similitudes : il était alors doctorant en mathématiques, sous la direction de Laurent Schwartz. Or, ce dernier est en partie à l'origine du Comité Maurice Audin. De même, René de Possel, dont Maurice Audin a été l'assistant, a l'idée d'organiser la soutenance de sa thèse in abstentia en décembre 1957, moment fort de dénonciation et de médiatisation du sort du jeune disparu ${ }^{17}$.

Par la suite, le relais dans les milieux universitaires ou intellectuels s'élargit en dépassant le cadre des connaissances personnelles et, globalement, la mobilisation des universitaires repose sur d'autres motivations que l'existence d'un lien personnel avec une victime de la torture. Mais ce lien personnel semble indispensable au départ pour vaincre l'incrédulité et le doute, inspirer la confiance, provoquer l'engagement. Cette nécessité d'une garantie de la vérité du témoignage est essentielle. C'est la conséquence directe de la dissimulation entreprise. Dans la même logique, pour emporter l'adhésion et convaincre l'opinion publique, alors que les auteurs de torture organisent la dissimulation de leurs actes, la notoriété et la respectabilité des personnes engagées servent de caution. Ainsi s'explique la place des universitaires dans le cercle de ceux qui s'engagent.

A contrario, les difficultés auxquelles se heurtent les communistes témoignent de la défiance que



Page de couverture de La question, d'Henri Alleg, publié par Jérôme Lindon aux Editions de Minuit en février 1958. (Coll. BDIC)

peut susciter la divulgation de la pratique de la torture. Les communistes publient de façon régulière des témoignages de tortures. La chronologie des affaires révélées dans l'Humanité diverge de la chronologie générale dans la mesure où les premières révélations sont précoces. De plus, les communistes sont très actifs dans la dénonciation de la torture : avocats, militants du PCA, militants métropolitains engagés dans des Comités contre la guerre... Mais, souvent, les affaires prises en charge par le seul réseau communiste sont entachées par un manque de crédibilité aux yeux de l'opinion. L'affaire dite des " torturés d'Oran »le démontre. Il s'agit d'une dizaine de militants communistes, syndicalistes et sympathisants arrêtés en septembre 1956 à Oran par la DST. D'une grande envergure, l'affaire occupe toute la presse pendant plus d'un mois au point qu'une Commission d'enquête parlementaire est formée, mais à une condition : qu'elle ne compte pas de députés communistes en son sein. La présence de communistes aurait fait perdre de la crédibilité à la Commission d'enquête. Cet exemple illustre les difficultés que doivent surmonter les militants engagés dans la dénonciation de la torture, la vraisemblance des faits se mesurant à l'aune de leur image dans l'opinion publique.
16. Cf. le rapport de Maurice Garçon, publié par Pierre Vidal-Naquet dans La raison d'État (Paris, Minuit, 1962, pp. 129 à 167), et le témoignage de Louis Martin-Chauffier, " Journal en marge d'une enquête ", paru dans la revue Saturne $\left(n^{\circ} 15\right.$, octobre-novembre 1957, p. 8).

17. Cf. le tome 2 des Mémoires de Pierre VidalNaquet, Paris, La Découverte, 1998, pp. 61-63. 
Les milieux chrétiens, eux aussi très engagés dans la dénonciation de la torture, ne rencontrent pas ce type de problème. Ils trouvent assez facilement une audience plus large, usant de leurs relais dans les milieux intellectuels. Le tout premier livre dénonçant la torture, intitulé Contre la torture, paru en mars 1957 aux éditions du Seuil, a ainsi été écrit par Pierre-Henri Simon, membre du Cercle des écrivains catholiques.

La notoriété et la respectabilité de ceux qui s'engagent jouent d'autant plus qu'il leur faut vaincre les réticences de ceux qui publient : les journalistes. Dans ce dernier cercle entre les victimes et l'opinion, deux éléments freinent considérablement la révélation d'affaires de tortures. La peur de la saisie, d'abord : $35 \%$ des saisies concernent en effet les seules affaires Alleg, Audin et Boupacha, trois affaires de dénonciation de la torture. En l'absence de censure préalable, la saisie a posteriori est une arme très efficace pour le pouvoir, en raison des pertes financières qu'elle entraîne, les exemplaires saisis ayant été imprimés mais non vendus. La presse fait donc preuve de prudence avant de se lancer dans la révélation d'une affaire et tend à pratiquer l'autocensure. D'après Jérôme Lindon, directeur des éditions de Minuit, les éditeurs ont dû pallier les " carences » de la presse en prenant la décision de publier certains récits quand la presse, timorée, se refusait à le faire ${ }^{18}$. Les éditions de Minuit et les éditions Maspero, créées en 1959 pour publier des écrits favorables au FLN, ont ainsi assuré la publication d'un nombre important de livres dénonçant la répression. De même, l'année 1957, année de la révélation, voit se multiplier les livres et les brochures publiés par d'autres canaux que ceux de la grande presse métropolitaine.

Le deuxième frein à la dénonciation de la torture par les journalistes est leur appréciation de la situation politique au moment où ils reçoivent des témoignages. Pour eux, non seulement le récit doit être vrai, authentifié, certifié par ceux qui le leur transmettent, mais de plus, sa révélation ne doit pas être inopportune. C'est ainsi que JeanMarie Domenach a reçu de nombreux témoignages de rappelés en 1956. Mais il décide de ne pas les publier. Par contre, il écrit au colonel Lacheroy, responsable de l'action psychologique, pour l'informer des violences commises par les troupes françaises en Algérie et l'inciter à réagir. II ne reçut pour toute réponse que le mépris : "L'autre le traita de boy-scout", raconte Pierre Vidal-Naquet ${ }^{19}$. L'attitude de Jean-Marie Domenach s'explique par sa réticence à gêner le gouvernement en rendant publique une dénonciation de la répression. Pour Jean-Marie Domenach, il faut laisser le temps au gouvernement de Guy Mollet, qui a défini sa politique algérienne par le triptyque " cessez-le-feu, élections, négociations ", de la mettre en œuvre. De son côté, Hubert Beuve-Méry résume bien cette difficile responsabilité du journaliste partagé entre son devoir d'informer et la peur de mettre dangereusement en difficulté le pouvoir, dans l'éditorial qu'il consacre à la sortie de Contre la torture de Pierre-Henri Simon : "Témoins ou victimes d'atrocités commises en Algérie par des Français, des lecteurs s'affligent de la relative discrétion qu'observe Le Monde à se sujet. Pour d'autres au contraire, toute information qui peut être exploitée contre nous est déjà une trahison. Le dilemme est redoutable $" 20$. La peur de trahir et de gêner un gouvernement explique donc la longue plage de silence sur les tortures dans la presse, de janvier 1955 à la fin de I'hiver 1957, exceptée l'affaire des torturés d'Oran qui éclate à l'automne 1956

A partir de mars 1957, cette peur de gêner un gouvernement ou de trahir son pays saute sous l'avalanche des affaires liée à une modification du contexte : d'abord, cette année 1957 s'ouvre sur la bataille d'Alger, marquée par un quadrillage systématique, une multiplication des arrestations donc des interrogatoires et, mécaniquement, de la torture. De plus, celle-ci est justifiée par le commandement qui voit en elle une méthode efficace pour obtenir des renseignements et détruire le FLN. Par ailleurs, en 1957, l'opinion publique est peut-être plus réceptive aux récits dénonçant les formes prises par la répression en Algérie. C'est que le compte rendu quotidien de la guerre d'Algérie s'enfle, se nourrit de récits sur la répression tandis que la politique du gouvernement Guy Mollet a fait ses preuves. Face à l'enfoncement dans la guerre, l'opinion prête une oreille plus attentive aux dénonciations des violences commises au nom de la France. Enfin, à partir de 1957, les témoignages de rappelés abondent. La plupart du temps, ceux-ci parlent à leur retour. Or, les premiers retours de rappelés envoyés en Algérie s'effectuent en 1957. C'est ainsi que «La paix des Nemenchtas ", de Robert Bonnaud, est publié dans Esprit en avril 1957, au retour de cet agrégé $d$ 'histoire envoyé en Algérie. Comme les autres, les récits des rappelés doivent émaner de personnes de confiance, avec une garantie de la véracité du témoignage et, dans le cas de "La paix des Nemenchtas ", la seule qualité d'agrégé d'histoire de son auteur suffit à cautionner ses propos : "Je ne pouvais imaginer que ce témoignage écrit par un homme qui était comme moi, un professionnel de la vérité (c'est-à-dire un agrégé d'histoire!) et qui signerait de son nom, ne soulèverait pas un immense scandale ", témoigne Pierre VidalNaquet ${ }^{21}$. Le dossier Jean Müller, supplément au numéro 38 de Témoignage chrétien, qui paraît en février 1957, offre également des garanties par la personnalité de son auteur. En effet, Jean Müller a été tué en octobre 1956 et son "dossier " rassemble les lettres qu'il avait envoyées à son frère. Les textes publiés n'ont donc pas été écrits dans l'objectif d'être publiés et de provoquer le scandale. Mais surtout, Jean Müller était permanent de l'Action catholique, membre des Scouts de France et quatorze témoins retrouvés par son frère sont prêts à témoigner de la véracité des faits qu'il relate.

Après la rupture de mai 1958, sous la Cinquième République, peu d'affaires retiennent de nouveau l'attention de l'opinion. Seules trois grandes affaires marquent cette période : La gangrène aux éditions de Minuit, en juin 1959; les deux volumes du Cahier vert des disparitions en Algérie, à l'automne $1959^{22}$; I'affaire Djamila premier en octo

1959 et le deuxième en novembre. 
Boupacha qui éclate en mai 1960. Pourtant, la pratique de la torture ne cesse pas.

La première explication de ce décalage est que la conjoncture de la Cinquième République est assez identique à celle de l'année 1956. Après 1958 , la presse fait de nouveau preuve d'attentisme, tant l'espoir d'un tournant politique de grande ampleur est fort. La rupture a été promise par André Malraux en juin 1958 : «Aucun acte de torture ne s'est produit à ma connaissance, ni à la vôtre, depuis la venue à Alger du général de Gaulle. Il ne doit plus s'en produire désormais $" 23$. Cette déclaration marque la reconnaissance par les plus hautes autorités de l'État de la pratique de la torture. De plus, comme une modification des pratiques est annoncée, la presse attend les résultats des bouleversements politiques. La première grande affaire, La gangrène, éclate d'ailleurs pour prouver que la promesse d'André Malraux n'a pas été tenue.

Les dénonciations d'affaires de torture se ralentissent également en raison de la personnalité et de la politique du général de Gaulle qui suscitent le ralliement $d$ 'intellectuels autrefois engagés dans la dénonciation de la torture, comme Pierre-Henri Simon ou François Mauriac. Enfin et surtout, le temps de la révélation est passé. Pour ceux qui ont contribué à percer le secret de la pratique de la torture, à le médiatiser, à emporter l'adhésion d'une partie de l'opinion et des intellectuels, audelà de 1957, l'essentiel est d'entretenir l'information. Certes, le Comité Maurice Audin continue à publier des témoignages. Mais, pour ce Comité, le temps de la preuve au sens juridique du terme succède à celui de la révélation : le Comité porte ses efforts sur l'instruction de la plainte déposée après la disparition de Maurice Audin, dont il obtient le transfert en métropole, à Rennes, en avril 1959. Puis il se lance dans un procès en diffamation contre La voix du Nord en mai 1960.

Mais après les révélations de 1957, il devient difficile de créer de nouveau un scandale car la pratique de la torture est connue. La question qui se pose désormais est de savoir ce que fait le général de Gaulle contre la torture, surtout après les assurances d'André Malraux. Par conséquent, les affaires ont pour objectif essentiel $d^{\prime}$ interpeller le nouveau gouvernement, la rupture promise n'ayant pas lieu. La gangrène s'ouvre ainsi sur une citation d'Edmond Michelet, ministre de la justice : "Il s'agit là de séquelles de la vérole, du tota- litarisme nazi ". De même, le cas de Djamila Boupacha est présenté à la presse en mai 1960 accompagné de cette anecdote : le père de Djamila aurait crié aux militaires "De Gaulle a dit qu'on ne torturerait plus " et le militaire qu'il avait en face de lui lui aurait répondu "De Gaulle, qu'il fasse ce qu'il veut chez lui, ici, c'est nous qui commandons $"{ }^{24}$.

Enfin, dernière preuve que le temps de la révélation est passée, on entre dans une période de réflexion sur de possibles inculpations pour crime contre l'humanité incluant la torture. En 1961, Nuremberg pour l'Algérie, de Maîtres Ben Abdallah, Oussedik et Vergès, aux éditions Maspero, en fait la tentative. Sous la Cinquième République commence donc la construction d'un objet juridique, par la recherche d'une qualification pour le crime qu'est la torture commise par les forces de l'ordre françaises œuvrant en Algérie.

Il n'a pas fallu attendre longtemps pour que le secret de la torture soit divulgué. Pendant la guerre, les dénonciations de cette pratique ont été foison et ces informations, portées à la connaissance de l'opinion publique pour des motifs divers, constituent aujourd'hui encore une source pour I'historien. La dissimulation de la pratique de la torture a donc été relativement inefficace. Grâce aux témoignages qui abondent, aux livres qui existent, la pratique de la torture en Algérie pendant la guerre est incontestable. Le mérite des opposants à la torture réside dans cette production de sources.

Les stratégies de dissimulation n'ont pas pour autant échoué totalement. Pour répondre aux nombreuses questions qui demeurent autour de la torture, les archives sont rarement explicites et I'historien doit composer avec les différents voiles placés sur la réalité par les contemporains, au risque de se heurter parfois à l'incrédulité que suscitent le décryptage des euphémismes, périphrases et silences des documents écrits, ainsi que le recours aux témoignages oraux. Les stratégies de dissimulation continuent donc de produire leurs effets par ces handicaps pour l'historien.

Raphaëlle BRANCHE ATER à l'Université de Reims

Sylvie THENAULT Docteur en histoire
23. Le Monde, 26 juin 1958 , p. 3.

24. Cité notamment par Le Monde, édition du 27 mai 1960 , page 5 . 